\title{
A Binary Matrix Method to Enumerate, Hierarchically Order and Structurally Classify Peptide Aggregation
}

Amol Tagad (orcid.org/0000-0002-4004-1638)

Reman Kumar Singh* (orcid.org/0000-0001-6160-4586)

G. Naresh Patwari* (orcid.org/0000-0003-0811-7249)

Department of Chemistry, Indian Institute of Technology Bombay, Powai, Mumbai 400076 INDIA.

*Email: reman53@gmail.com (RKS); naresh@chem.iitb.ac.in (GNP)

\begin{abstract}
Protein aggregation is a common and complex phenomenon in biological processes, yet a robust analysis of this aggregation process remains elusive. The commonly used methods such as centre-of-mass to centre-of-mass (COM-COM) distance, the radius of gyration $\left(R_{g}\right)$, hydrogen bonding (HB) and solvent accessible surface area (SASA) do not quantify the aggregation accurately. Herein, a new and robust method that uses an aggregation matrix (AM) approach to investigate peptide aggregation in a MD simulation trajectory is presented. A $n x n$ twodimensional aggregation matrix (AM) is created by using the inter-peptide $C_{\alpha}-C_{\alpha}$ cut-off distances which are binarily encoded ( 0 or 1 ). These aggregation matrices are analysed to enumerate, hierarchically order and structurally classify the aggregates. Moreover, the comparison between the present AM method and the conventional $R_{g}, C O M-C O M$ and $\mathrm{HB}$ methods shows that the conventional methods grossly underestimate the aggregation

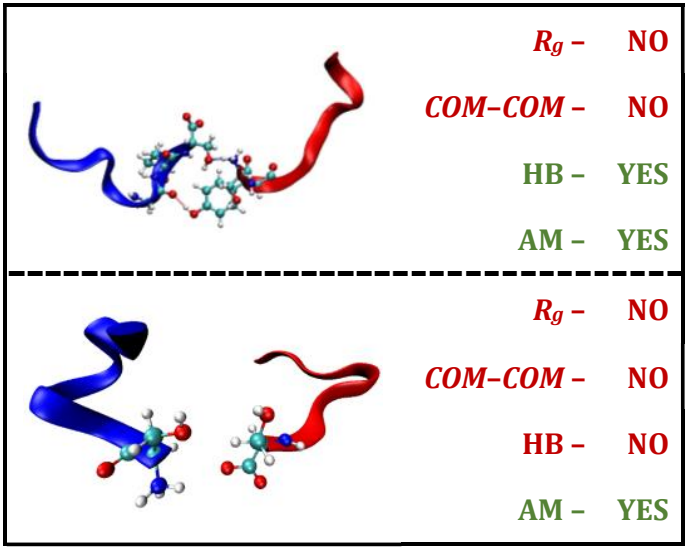

propensity. Additionally, the conventional methods do not address the hierarchy and structural ordering of the aggregates, which the present AM method does. Finally, the present AM method utilises only $n \times n$ two-dimensional matrices to analyse aggregates consisting of several peptide units. To the best of our knowledge, this is a maiden approach to enumerate, hierarchically order and structurally classify peptide aggregation.
\end{abstract}




\section{INTRODUCTION}

Protein-protein (or inter-protein) interactions are important steps for many biological processes, ${ }^{1-3}$ such as translation, ${ }^{4}$ transcription factor, ${ }^{5}$ cell signalling, ${ }^{6-8}$ protein synthesis, 9,10 and many others. These inter-protein interactions are primarily driven by high hydrophobicity, low charge density, and high $\beta$-sheet propensity. ${ }^{11,12}$ Many interprotein interactions involve folded proteins, however, in some cases, these interactions are formed by disordered or structureless protein/peptides which aggregate to form fibrillar structures and result in amyloid fibrils. ${ }^{13-17}$ These aggregates are organized as cross $\beta$-sheet patterns, where the $\beta$-sheet backbone is perpendicular to the fibril axis ${ }^{8,20}$ and are insoluble. ${ }^{18-20}$ These protein aggregates are associated with diseases like Alzheimer's, ${ }^{21-23}$ Parkinson's, ${ }^{16,24,25}$ Huntington's, type II diabetes mellitus, ${ }^{7,24,25}$ and several others. Therefore, the atomic level understanding of the aggregation process has become more important. Apart from several experimental methodologies, $2,16,26-28$ molecular dynamics (MD) simulations have been frequently used to investigate peptide and protein aggregation to understand the aggregation pathways along with their structural characterization and dynamic behaviour. In this regard, several physical and chemical parameters such as centre-of-mass to centre-of-mass (COM-COM) distance, the radius of gyration $\left(R_{g}\right)$, the dihedral angle between the peptide chains, hydrogen bonding (HB), solvent accessible surface area (SASA), and contact matrix have been primarily used to probe the aggregation in a MD trajectory. ${ }^{22,29,30}$ While the above mentioned methods have been utilized to investigate peptide/protein aggregation, the global methods such as COM-COM, $R_{g}$ and dihedral angle between the peptide chains do not consider the residue level interaction. On the other hand, the residue level methods such as HB, SASA and contact matrix are in general qualitative and not quantitative. More importantly, none of these methods, except the HB method, provide information on the hierarchy of aggregation, which can be utilised to estimate the propensity to form compact and energetically favourable aggregates. Furthermore, none of these methods including HB can differentiate the structurally ordering, such as the formation of parallel and antiparallel aggregates. Herein, a new and robust method, which uses an aggregation matrix approach to investigate peptide aggregation in a MD simulation trajectory. This method utilizes the inter-peptide $C_{\alpha}-C_{\alpha}$ cut-off distances which are binarily encoded (0 or 1) to create $n \times n$ two-dimensional matrices, which are further analysed to enumerate, hierarchically order and structurally classify the aggregates. In the present work, the 
aggregation propensity of six known low complexity domain (LCD) peptides, 11,19,31-40 using the aggregation matrix approach is investigated and compared with the other methods.

\section{METHODOLOGY}

In this work, six LCD peptides with the sequences NKGAII (3Q2X), ${ }^{41}$ STGGYG (6BZP), ${ }^{2}$ SYSGYS (6BWZ), ${ }^{2}$ SYSSYGQS (6BXV), ${ }^{2}$ GYNGFG (6BXX), ${ }^{2}$ and GFGNFGTS (6BZM) ${ }^{2}$ were considered. The initial conformation was taken directly from the PDB and the dimers, trimers and tetramers were generated by replicating the monomers and placing them such that the surface area of the monomers is at a distance of $10 \AA$ in a cubic box with the dimension such that the peptide does not interact with its periodic image during the simulation. The peptides were solvated by TIP3P water model ${ }^{42}$ and $\mathrm{Na}^{+}$and $\mathrm{Cl}^{-}$ions were added to neutralize the system. All the simulations were carried out with CHARMM36 force field ${ }^{43}$ using GROMACS 2019 software package. ${ }^{44}$ The potential energy of the system was minimized for 5000 steps using the steepest descent method.45 During the minimization a $10 \AA$ A cut-off was used for van der Waals and electrostatic interactions. Particle mesh Ewald (PME) method was used to model the electrostatic interactions. ${ }^{46}$ Additionally, the bonds were constrained using the LINCS algorithm. ${ }^{47}$ Following energy minimization, each system was equilibrated in NVT and NPT ensembles for 200 ps and 1000 ps, respectively. ${ }^{48}$ The velocity rescaling thermostat ${ }^{49}$ and ParrinelloRahman barostat ${ }^{50}$ were used to maintain $300 \mathrm{~K}$ temperature and 1 bar pressure with a coupling constant of 0.4 ps. Finally, the production simulation using NPT condition at 300 $\mathrm{K}$ and 1 bar pressure was carried and the equations of motion were integrated using the leapfrog algorithm with a time step of 2 fs. The simulation time was $2 \mu$ s for each of the systems containing two, three and four peptide units, and the coordinates were saved every 10 ps during the simulation and the data was further analysed.

The formation of the peptide aggregates was analysed using inter-peptide pairwise $C_{\alpha}-C_{\alpha}$ distances. The inter-peptide $C_{\alpha}-C_{\alpha}$ distances were cast as binary logic of 1 or 0 with a cut-off distance criterion and an aggregation matrix was generated. The entries in the aggregation matrix would be 1 or 0 depending on whether the inter-peptide $C_{\alpha}-C_{\alpha}$ distance was less than or equal or greater to the cut-off distance, respectively. For a $n$ - 
mer peptide the aggregation matrix would be of the order $n \times n$ with the matrix elements being 0 or 1 . The advantage of using an aggregation matrix is that each element $a_{i j}$ encodes the inter-peptide interaction between the $C_{\alpha}$ of $i^{\text {th }}$ residue of one (A) peptide to the $C_{\alpha}$ of the $j^{\text {th }}$ residue of the other (B) peptide. The aggregation matrices were generated for each snapshot over the entire trajectory.

The formation of an aggregated dimer can be defined when the sum of all the elements of the aggregation matrix, the aggregation number, which is defined as

$$
\sum_{i=1}^{n} \sum_{j=1}^{n} a_{i j}
$$

must be non-zero. The aggregation number can also be used to define the hierarchy of aggregation, wherein a larger aggregation number indicates a more compact structure. Further, the formation of a specific type of aggregate can also be inferred from the aggregation matrix. For instance, the formation of a quasi-parallel dimer configuration can be recognized by the sum of the diagonal elements, which is defined as

$$
\sum_{i=1}^{n} \sum_{j=i}^{n} a_{i j}
$$

On the other hand, a quasi-anti-parallel dimer configuration can be recognized by the sum the anti-diagonal elements, which is defined as

$$
\sum_{i=1}^{n} \sum_{j=n+1-i}^{n} a_{i j}
$$

the sum of the diagonal/anti-diagonal elements being greater than or equal to 3 for the formation of parallel/anti-parallel strands. The choice of minimum value of 3 for such classification is based on the fact that a lower value would not be able to distinguish between the parallel/anti-parallel structures at marginally higher $C_{\alpha}-C_{\alpha}$ cut-off distances and a very small $C_{\alpha}-C_{\alpha}$ cut-off distances must have to be employed, which might artificially lower the aggregation propensity. Similarly, several shifted parallel and shifted anti-parallel dimer configurations can be defined by either increasing or decreasing the index $j$ relative to index $i$, such as

$$
\sum_{i=1}^{n} \sum_{j=i \pm k}^{n} a_{i j} \text { where }|n-k| \geq 3
$$

for shifted parallel, and

$$
\sum_{i=1}^{n} \sum_{j=n+1-i \pm k}^{n} a_{i j} \text { where }|n-k| \geq 3
$$


for shifted anti-parallel configurations.

A trimer has three identical peptides, however, for representation purposes three of the peptides are indexed as A, B, and C. In a system with three peptides, two modes of aggregation are feasible, a dimer and a trimer. Even in the case of a dimer, three independent modes of dimer formation are feasible which can be characterized by $\mathrm{AB}, \mathrm{BC}$ and CA dimers. To begin with, all three sets of dimers (corresponding to $\mathrm{AB}, \mathrm{BC}$ and $\mathrm{CA}$ ) formation were analysed independently based on the aggregation number given in equation (1). A trimer formation is recognized by snapshots/frames that contain two or more independent dimers. The hierarchy in the trimers can be defined by the sum of elements in all the three independent dimer aggregation matrices. This methodology was extended to tetramers as well, in which six independent dimers $(A B, A C, A D, B C, B D$, and $\mathrm{CD}$ ), four independent trimers (ABC, $A B D, A C D$, and $B C D$ ) and a tetramer are formed. $A$ trimer is formed by snapshots/frames that contain two or more independent dimers with one common peptide unit (five out of six combinations will satisfy this condition). Finally, the formation of tetramers was based on the inter-peptide $C_{\alpha}-C_{\alpha}$ distance being less than the cut-off value across the three sets of mutually exclusive dimers (AB-CD, AC-BD, and $\mathrm{AD}-\mathrm{BC}$ ) in a single snapshot. Further, the propensity of form ordered structures such as parallel, shifted parallel, anti-parallel and shifted anti-parallel, for trimers and tetramers were analysed based on equations (2-5).

\section{RESULTS}

The aggregation behaviour of the six LCD peptides considered in the present work was analysed based on the aggregation matrix method, which was generated throughout the trajectory for several inter-peptide $C_{\alpha}-C_{\alpha}$ distances cut-off distances of $0.50,0.55,0.60$ 0.65 , and $0.70 \mathrm{~nm}$. Figure 1 shows the dimer population as a function of $C_{\alpha}-C_{\alpha}$ cut-off distance for all the peptides in a system containing two peptide units. The dimer population changes significantly with the change in cut-off distance in the 0.5 to $0.6 \mathrm{~nm}$ range, thereafter the population change is gradual. It can be noticed from Figure $1 \mathrm{~A}$ that the extent of dimer population differs with each sequence which suggests that the aggregation depends on the amino acid sequence. The peptides SYSSYGQS and STGGYG 


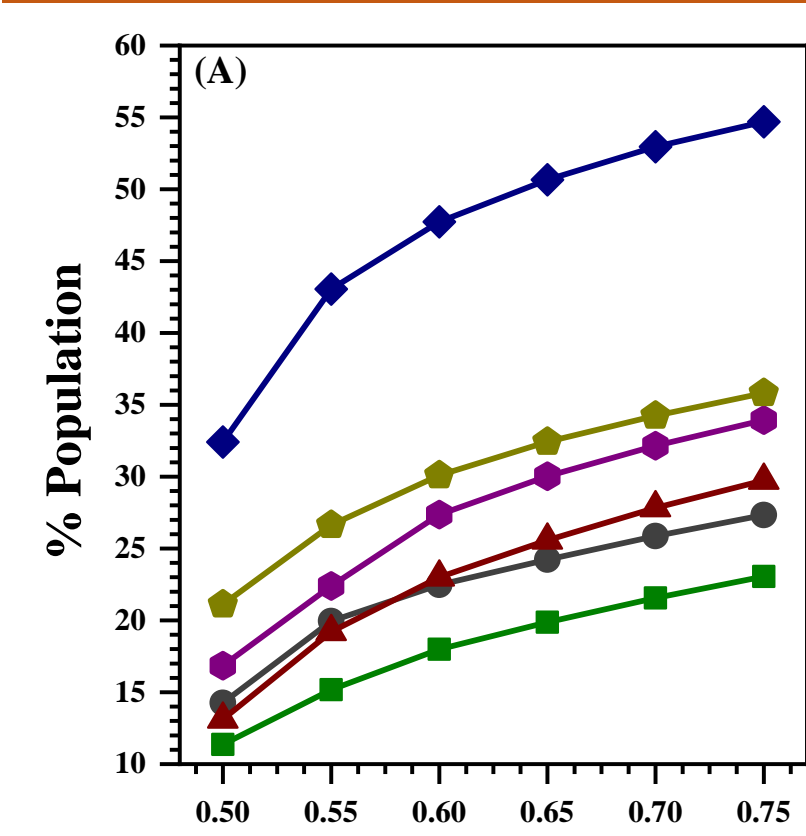

Cut-off Distance / nm

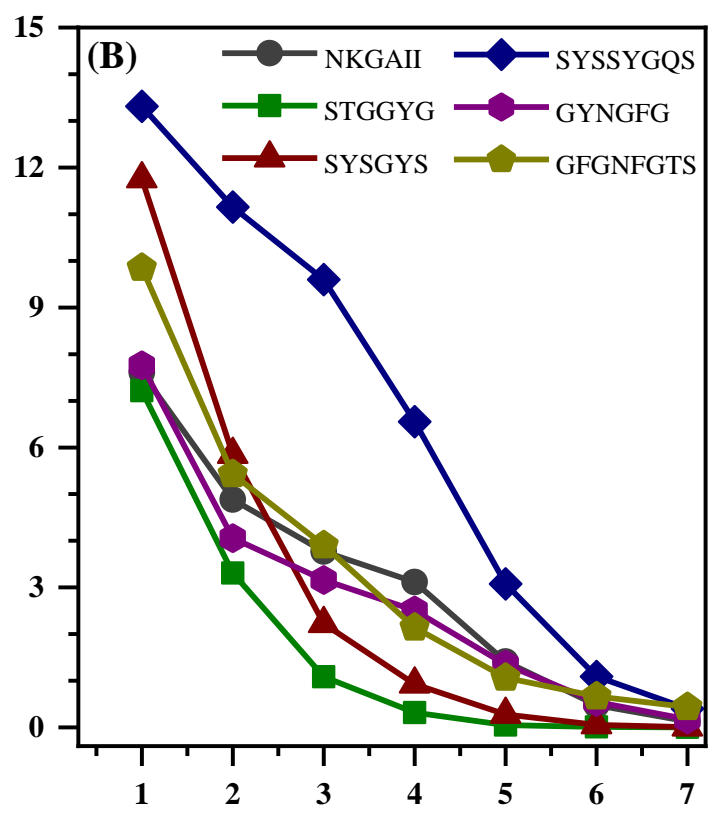

Number of Hydrogen Bonds

Figure 1. Dimer population (A) calculated using aggregation matrix as function of $C_{\alpha^{-}}$ $C_{\alpha}$ cut-off distance and (B) as a function of number of inter-peptide hydrogen bonds. Note that the legends in both the plots are same.

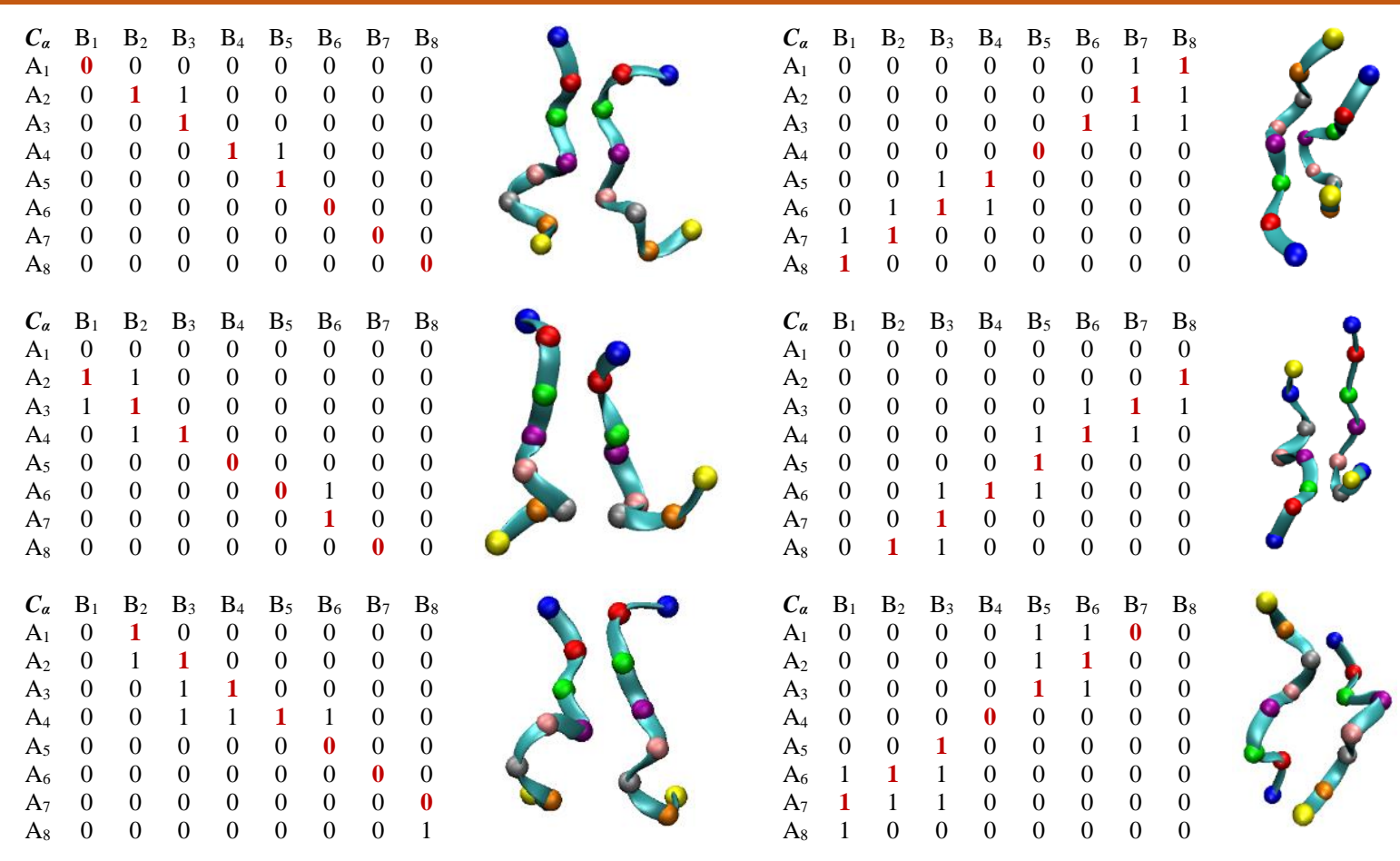

Figure 2. The matrix representations for the parallel/shifted parallel (left panel); antiparallel / shifted anti-parallel (right panel) configurations of the SYSSYGQS dimer with the $C_{\alpha}-C_{\alpha}$ cut-off distance of $0.6 \mathrm{~nm}$. Also shown are the structures corresponding to each matrix. 
Table 1: Representative dimer population (\%) along with the population of ordered and random configurations at various inter-peptide $C_{\alpha}-C_{\alpha}$ cut-off distances (nm).

\begin{tabular}{|l|ccc|ccc|}
\hline \multirow{2}{*}{ Cut-off } & \multicolumn{3}{|c|}{ NKGAII } & \multicolumn{3}{c|}{ SYSSYGQS } \\
\cline { 2 - 7 } & Dimer & Random & Ordered & Dimer & Random & Ordered \\
\hline 0.50 & 14.26 & 14.23 & 0.04 & 32.42 & 31.87 & 0.55 \\
\hline 0.55 & 19.94 & 19.45 & 0.49 & 43.06 & 37.52 & 5.54 \\
\hline 0.60 & 22.48 & 20.45 & 2.02 & 47.73 & 34.85 & 12.88 \\
\hline 0.65 & 24.24 & 20.10 & 4.14 & 50.65 & 32.44 & 18.22 \\
\hline 0.70 & 25.86 & 19.72 & 6.14 & 52.96 & 29.91 & 23.05 \\
\hline
\end{tabular}

have the highest and lowest propensity to form dimers, respectively, with the propensity to aggregate being higher for longer peptides. The formation of dimers was also analysed in terms of inter-peptide hydrogen bonds, shown in Figure 1B, and the results indicate very similar trends for various peptide sequences relative to the aggregation matrix method except for SYSGYS peptide, suggesting that the dimerization is primarily hydrogen bond driven. One of the important consequences of using the aggregation matrix method is its ability to hierarchically classify the structures based on the sum of all the entries in the aggregation matrix, the aggregation number. A larger aggregation number indicates formation of a more compact structure. Figure S1 (see the Supporting Information) shows the plots dimer population as a function of aggregation number, which shows the presence of bimodal distribution for three peptide sequences (NKGAII, SYSSYGQS and GFGNFGTS) and coarsely monotonic distribution for the other three peptides (STGGYG, SYSGYS and GYNGFG). Significantly, the propensity to form a compact dimer is maximum for the SYSSYGQS peptide, which is in line with the propensity of this peptide to dimerize. Further, the classification of aggregates as ordered and random can also be carried out using the aggregation matrix as elaborated in the methodology section and illustrated in Figure 2. In the present scenario, the ordering of peptides aggregates is based on the formation of parallel or anti-parallel dimers, which are common structural motifs in various $\beta$-sheet secondary structures of proteins. Additionally, Figure 2 also illustrates the formation of shifted parallel and shifted anti-parallel configurations. In the present analysis, all the parallel, anti-parallel, shifted parallel and shifted anti-parallel dimers are pooled as ordered aggregates while the remaining population can be considered as random (non-specific) aggregates. The total dimer population for the six peptides is listed in Table 1 and Table S1, along with their categorization into ordered and random aggregates. In general, the propensity to form random aggregates is higher 
than the ordered aggregates, however, within the ordered aggregates, the propensity to form anti-parallel / shifted anti-parallel configurations is higher than parallel / shifted parallel configurations. This observation is in excellent agreement with the favoured formation of antiparallel beta-sheets in peptide aggregates. ${ }^{2,31,51}$

In a system consisting of three peptides, the trimerization was analysed by decomposing the trajectory into three pairs of dimers and the formation of the trimer was
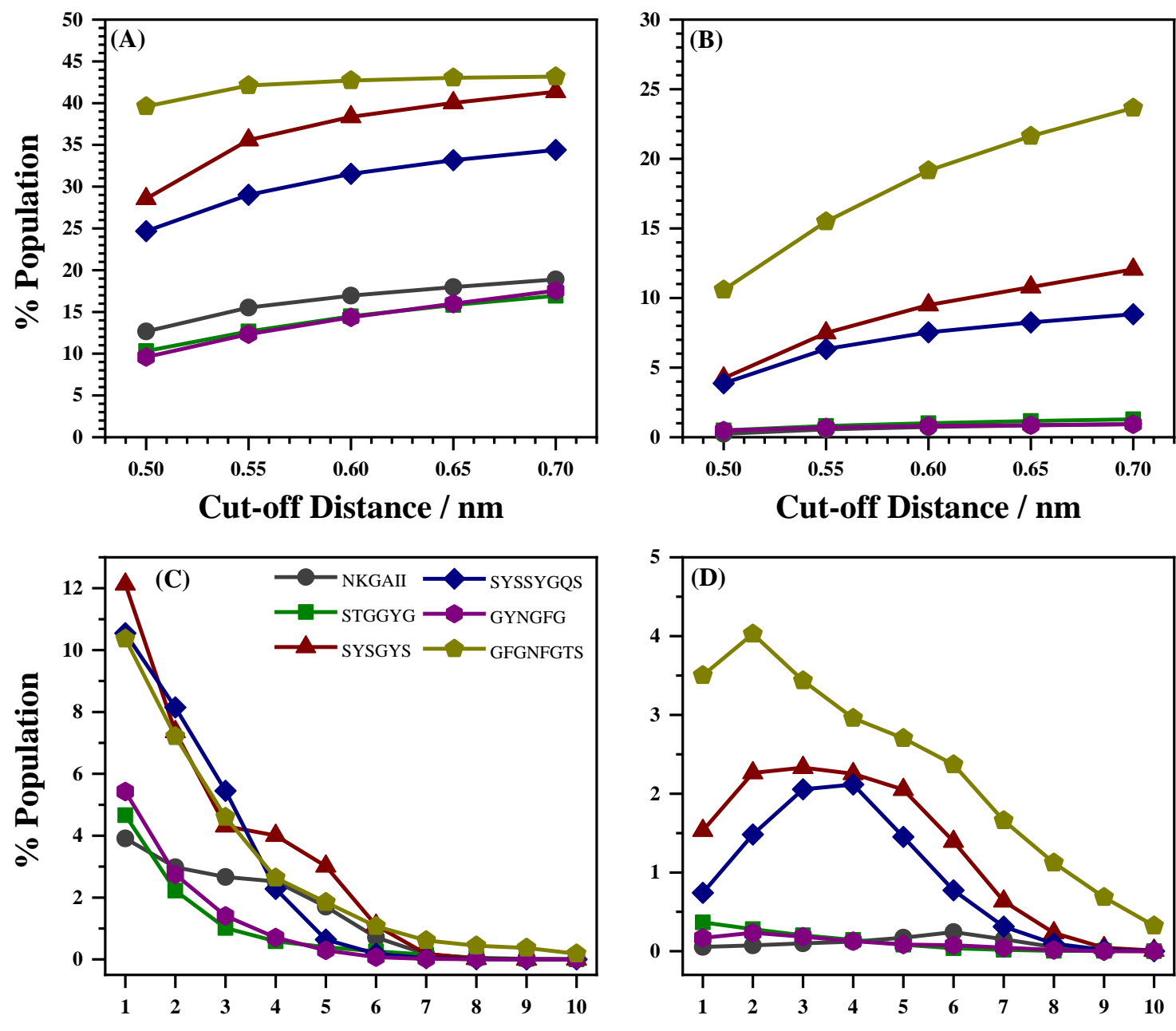

Number of Hydrogen Bonds

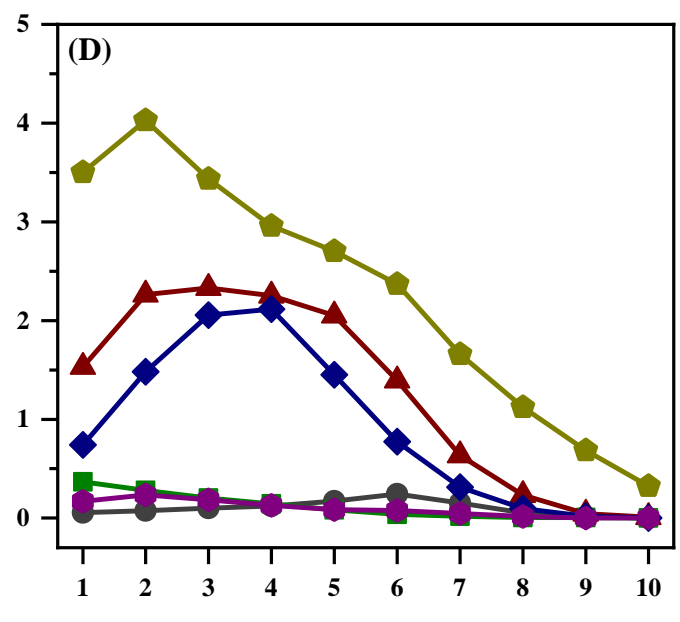

Number of Hydrogen Bonds

Figure 3. Population of (A) dimer and (B) trimers as function of $C_{\alpha}-C_{\alpha}$ cut-off distance in a system containing three peptides units. In $(A)$ the dimer population is the cumulative sum of all the three $\mathrm{AB}, \mathrm{BC}$ and $\mathrm{AC}$ dimers while removing common frames. In (B) the trimer population was evaluated by only considering the common frames for at least two dimers. Population of (C) dimers and (D) trimers as a function of number of inter-peptide hydrogen bonds. The population of dimers and trimers are mutually exclusive. Note that the legends in the all the plots are same. 
deciphered based on snapshots/frames with at least two sets of dimers. The population of the dimers and the trimers are mutually exclusive and are shown in Figure 3 as a function of $C_{\alpha}-C_{\alpha}$ cut-off distance. Interestingly, in the system containing three peptide units, the propensity to form either dimers or trimer showed a considerably different trend than the system containing two peptides. In this case, the sequence dependence on the propensity for trimer formation was GFGNFGTS > SYSGYS > SYSSYGQS and for the other three peptides, the timer population is negligible. Further, the aggregation behaviour was analysed in terms of inter-peptide hydrogen bonds, also shown in Figure 3 , and the results indicate very similar trends relative to the aggregation matrix method,

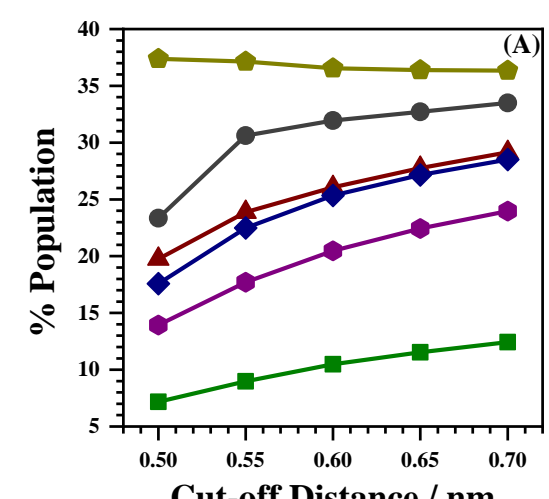

Cut-off Distance / nm

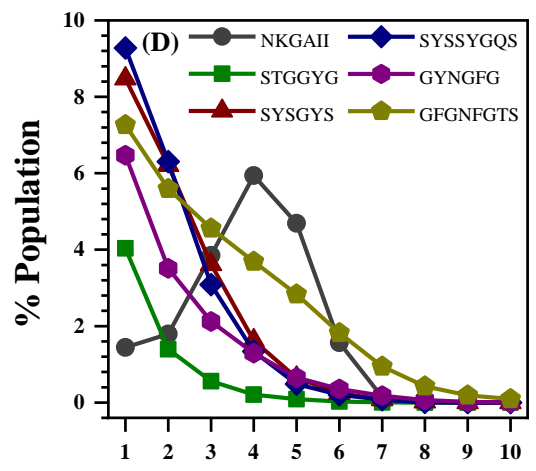

Number of Hydrogen Bonds

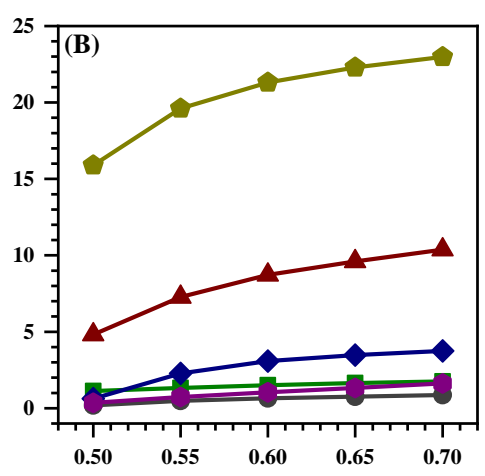

Cut-off Distance / nm

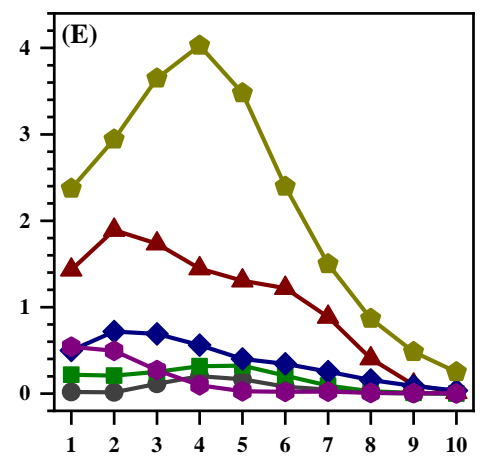

Number of Hydrogen Bonds

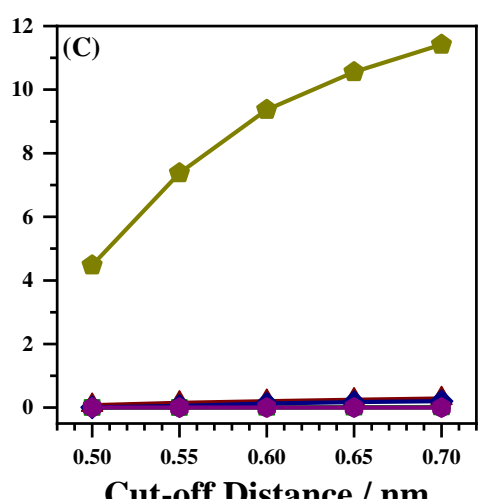

Cut-off Distance / nm

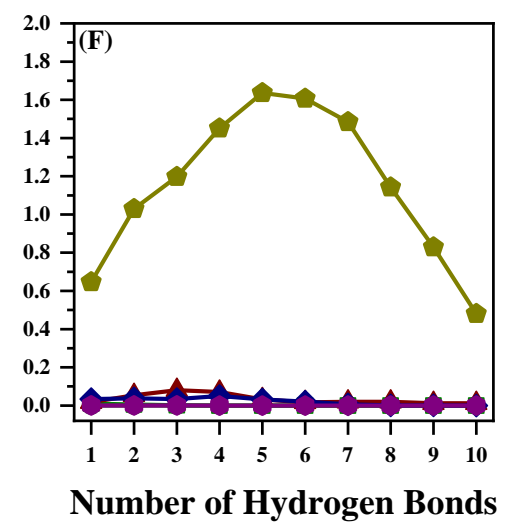

Figure 4. Population of (A) dimer, (B) trimers and (C) tetramers as function of $C_{\alpha}-C_{\alpha}$ cut-off distance in a system containing four peptides units. In $(A)$ the dimer population is the cumulative sum of all the six dimers $A B, A C, A D, B C, B D$ and $C D$ dimers while removing common frames. In (B) the trimer population was evaluated by only considering the common frames for at least two dimers with a common peptide unit (five out of six combinations will satisfy this condition), In (C) the tetramer formation evaluated based on the inter-peptide $C_{\alpha}-C_{\alpha}$ distance being less than the cut-off value across the three sets of mutually exclusive dimers (AB-CD, AC-BD, and AD-BC) in a snapshot. Population of (D) dimer, (E) trimers and (F) tetramers as a function of number of inter-peptide hydrogen bonds. The population of dimers, trimers and tetramers are mutually exclusive. Note that the legends in the all the plots are same. 
which once again suggests that the peptide aggregation is primarily hydrogen bond driven. Further, the hierarchy of aggregates was once again evaluated using the aggregation number (which is the sum of all the elements of the aggregation matrix) and the hierarchical ordering of the aggregates has the same sequence dependence as the propensity for aggregation (Figure S1, see the Supporting Information) both for the dimer and trimer formation. Finally, in a system containing four peptide units, the formation of dimers, trimers and tetramers were evaluated and the results are shown in Figure 4 as a function of $C_{\alpha}-C_{\alpha}$ cut-off distance. In this case, the aggregation trend is similar to the trimer and the aggregation matrix method, which once again shows a similar trend in hydrogen bonding. Even in this case, the hierarchical ordering of the aggregates has the same sequence dependence as the propensity for aggregation (Figure S1, see the Supporting Information) both for the dimer, trimer and tetramer formation. A twodimensional aggregation matrix approach to analyse the peptide aggregation in a MD

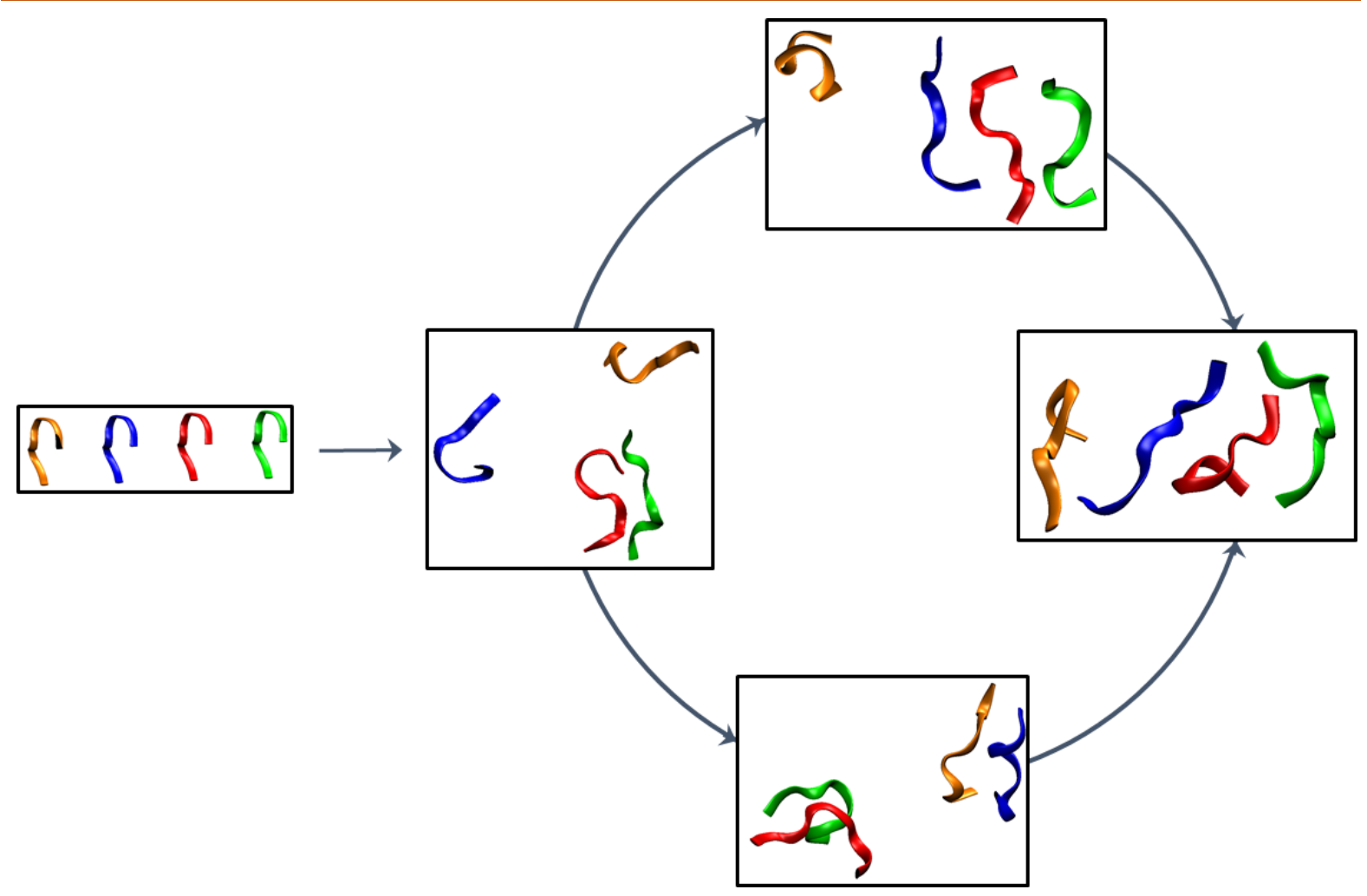

Figure 5. Snapshots of progression of aggregation in a system containing four peptide units of GFGNFGTS. Each peptide unit is colour coded for easy visualization. The formation of tetramer follows two pathways, first, step-wise addition, and second, dimerization of dimer. 
trajectory for systems consisting of up to four peptide units is a useful and convenient methodology. As noted earlier, this method is capable of enumerating, hierarchically ordering and structurally classifying the aggregates which can be illustrated in Figure 5, wherein the formation of tetramer can be mediated by sequential addition of a monomer to form dimer, trimer and tetramer. Alternatively, the formation of a tetramer can also be formed by the aggregation of two dimers.

\section{DISCUSSION}

It must be pointed out that the redundancy in the ordered aggregates increases with an increase in the $C_{\alpha}-C_{\alpha}$ cut-off distance as can be seen from Table $\mathrm{S} 1$, therefore caution must be exercised while interpreting the data corresponding to ordered aggregates. In view of the observed statistics, a $C_{\alpha}-C_{\alpha}$ cut-off distance of $0.55-0.60 \mathrm{~nm}$ is recommended.

Commonly used methods to characterize peptide aggregation in the literature use radius of gyration $\left(R_{g}\right)$ and centre-of-mass to centre-of-mass distance (COM-COM). ${ }^{22,29,30}$ Figure 6 shows the comparison of the present aggregation matrix method with the $R_{g}$ and COM-COM methods. The COM-COM method grossly underestimates the propensity of aggregation. On the other hand, the $R g$ method shows a comparable propensity of aggregation at a cut-off distance of $0.7 \mathrm{~nm}$ for the six-mer peptides, while the comparison for the eight-mer peptides shows substantial underestimation at the same cut-off distance of $0.7 \mathrm{~nm}$. Whilst the $R g$ method can be used to estimate the propensity of aggregation, the cut-off distance varies based on the length of the peptide and cannot be uniformly applied across all peptide sequences. Further, Figure 7 illustrates the formation of an end-to-end dimer for the SYSSYGQS peptide. In the two scenarios that are presented in Figure 7, both of them show very large values of $R_{g}$ and COM-COM, therefore are likely to be overlooked by both of these $\left(R_{g}\right.$ and $\left.C O M-C O M\right)$ methods. However, both these aggregates are enumerated by the aggregation matrix method even if the structure is devoid of any hydrogen bonds. It is, therefore, essential to realize that $R_{g}$ and COM-COM methods sample the global properties of the aggregate, while the aggregation matrix and hydrogen bonding methods sample the aggregation behaviour at the residue level. Moreover, the aggregation matrix method is also capable of capturing non-specific (read non-hydrogen-bonded) dimers. Additionally, both the $R_{g}$ and COM-COM methods are 

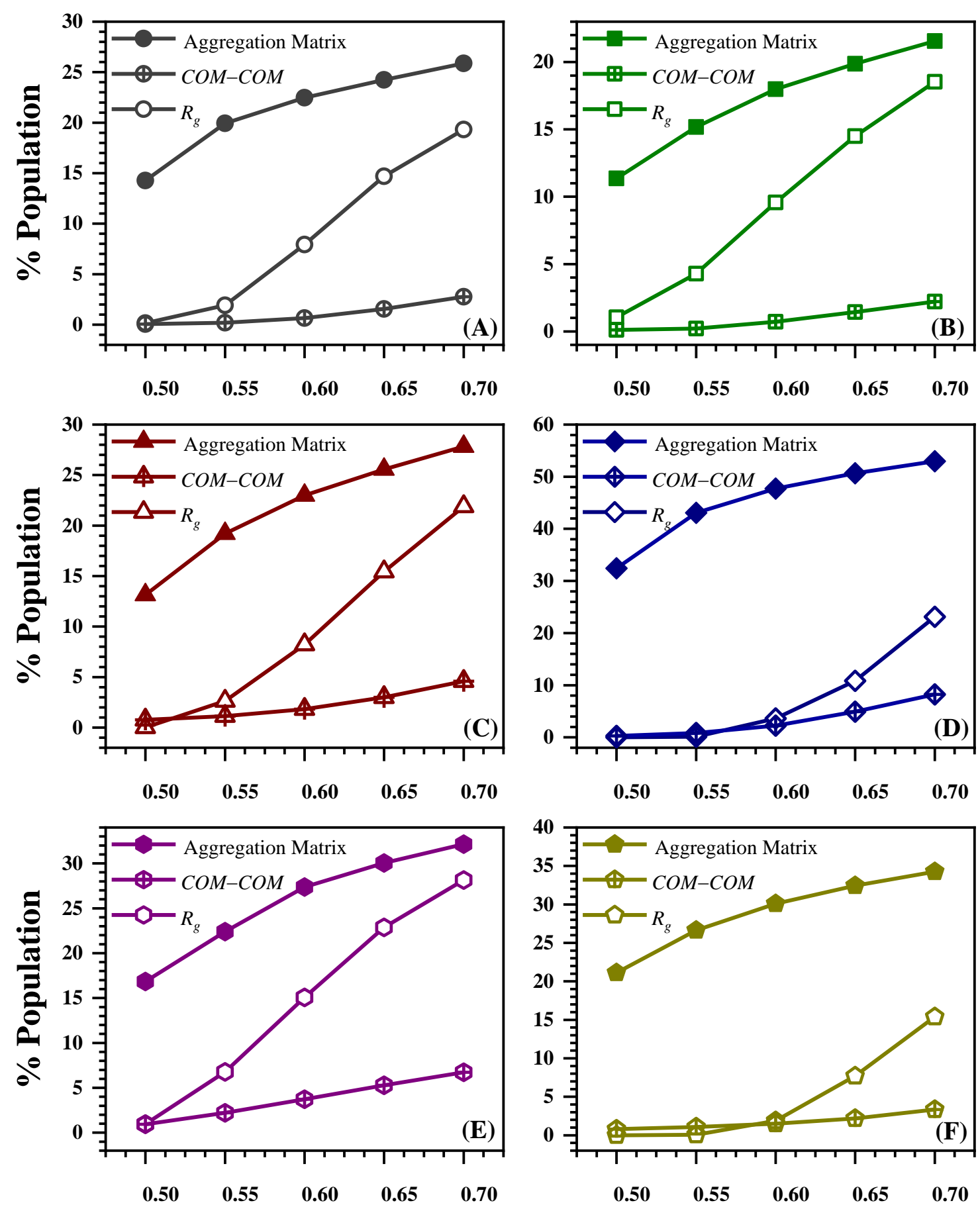

Cut-off Distance / nm

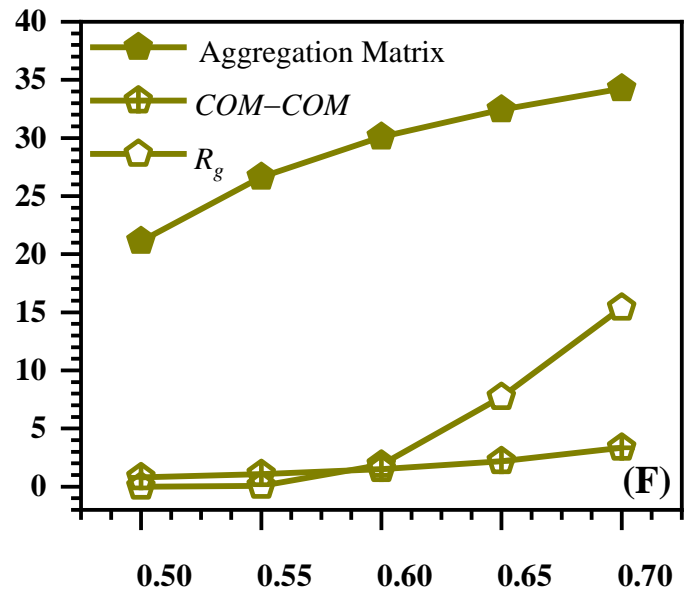

Cut-off Distance / nm

Figure 6. Comparison of dimer formation using the aggregation matrix method with methods radius of gyration $(R g)$ and centre-of-mass to centre-of-mass (COM-COM) methods for (A) NKGAII, (B) STGGYG, (C) SYSGYS, (D) SYSSYGQS, (E) GYNGFG, and (F) GFGNFGTS peptides. 
A

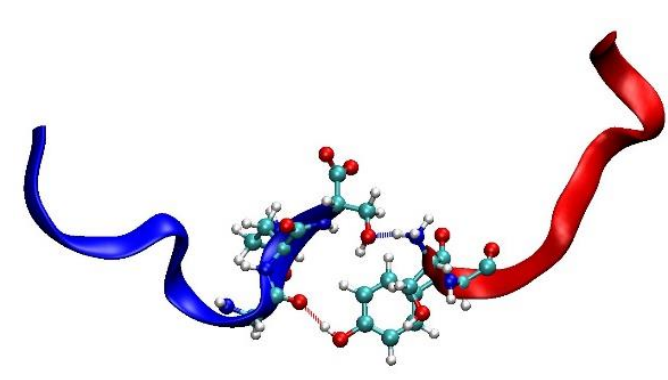

B

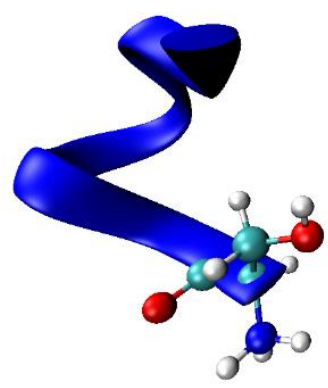

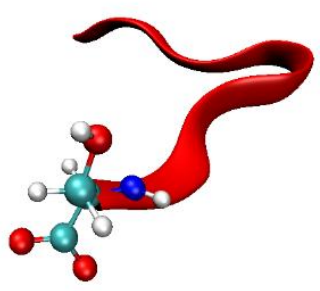

Figure 7. Formation of end-to-end SYSSYGQS dimer captured by the aggregation matrix method. For the dimer (A) the values of $R_{g}$ and COM-COM are 1.09 and $1.86 \mathrm{~nm}$, respectively, while the corresponding values of dimer (B) are 0.91 and $1.51 \mathrm{~nm}$, respectively. These structures might be overlooked depending on the cut-off values of $R_{g}$ used for analysis. The structure (A) shows the presence of two inter-peptide hydrogen bonds, while the structure (B) is a non-specific aggregate.

radially symmetric, therefore are not suitable for the hierarchical organization and structural classification (ordered vs. random) of peptide aggregates.

\section{CONCLUSIONS}

The aggregation propensity of six LCD peptides to form dimers, trimers and tetramers was examined using a newly developed aggregation matrix methodology. In this method an aggregation matrix is generated by considering the inter-peptide $C_{\alpha}-C_{\alpha}$ cut-off distances which are encoded to 0 and 1 depending on the distance cut-off. The total dimer population was obtained by counting the number of frames with an aggregation number of at least one. Further, the ordered conformations such as parallel/anti-parallel or shifted parallel/anti-parallel were obtained by analysing the diagonal/anti-diagonal and shifted diagonal/ani-diagonal, respectively. In a system consisting of three peptides units, the trimerization was analysed by decomposing the system into three pairs of dimers and the formation of the trimer was deciphered based on snapshots/frames with at least two sets of dimers. Similarly, the tetramer was analysed by decomposing the system into five dimers and tetramer formation was obtained when two mutually exclusive dimers are within cut-off distances. The explicit comparison of aggregation matrix with the conventional methods such as COM-COM distance and $R_{g}$ shows that the conventional methods underestimate the propensity of aggregation. Moreover, the conventional methods do not structurally classify structures such as parallel and anti-parallel, which 
the aggregation matrix method does by examining matrix elements. The present aggregation matrix is an easy and convenient method to analyse aggregation propensity in the MD trajectory which uses only nxn two-dimensional matrices even for systems consisting of several peptide units.

\section{Acknowledgments}

AT thanks University Grants Commission (UGC) for the research fellowship and RKS thanks IIT Bombay for the institute postdoctoral fellowship. Authors gratefully acknowledge the SpaceTime-2 supercomputing facility at IIT Bombay for the computing time. The support and the resources provided by 'PARAM Brahma Facility' under the National Supercomputing Mission, Government of India at the Indian Institute of Science Education and Research (IISER) Pune are gratefully acknowledged.

\section{Supporting Information}

The Supporting Information is available.

Plots showing Hierarchy of aggregation and tables containing population data of ordered and random configurations (PDF).

\section{References}

(1) Guenther, E. L.; Cao, Q.; Trinh, H.; Lu, J.; Sawaya, M. R.; Cascio, D.; Boyer, D. R.; Rodriguez, J. A.; Hughes, M. P.; Eisenberg, D. S. Atomic Structures of TDP-43 LCD Segments and Insights into Reversible or Pathogenic Aggregation. Nat. Struct. Mol. Biol. 2018, 25, 463-471.

(2) Hughes, M. P.; Sawaya, M. R.; Boyer, D. R.; Goldschmidt, L.; Rodriguez, J. A.; Cascio, D.; Chong, L.; Gonen, T.; Eisenberg, D. S. Atomic Structures of Low-Complexity Protein Segments Reveal Kinked $\beta$ Sheets That Assemble Networks. Science 2018, 359, 698-701.

(3) Zhou, C.; Sczepanski, J. T.; Greenberg, M. M. Histone Modification via Rapid Cleavage of C4'-Oxidized Abasic Sites in Nucleosome Core Particles. J. Am. Chem. Soc. 2013, $135,5274-5277$.

(4) Gallie, D. R. Protein-Protein Interactions Required during Translation. Plant Mol. Biol. 2002, 50, 949-970. 
(5) Alves, M. S.; Dadalto, S. P.; Gonçalves, A. B.; de Souza, G. B.; Barros, V. A.; Fietto, L. G. Transcription Factor Functional Protein-Protein Interactions in Plant Defense Responses. Proteomes 2014, 2, 85-106.

(6) Kirouac, D. C.; Saez-Rodriguez, J.; Swantek, J.; Burke, J. M.; Lauffenburger, D. A.; Sorger, P. K. Creating and Analyzing Pathway and Protein Interaction Compendia for Modelling Signal Transduction Networks. BMC Syst. Biol. 2012, 6, 29.

(7) Kuzmanov, U.; Emili, A. Protein-Protein Interaction Networks: Probing Disease Mechanisms Using Model Systems. Genome Med. 2013, 5, 1-12.

(8) Rutishauser, U.; Sachs, L. Cell-to-Cell Binding Induced by Different Lectins. J. Cell Biol. 1975, 65, 147-157.

(9) Marianayagam, N. J.; Sunde, M.; Matthews, J. M. The Power of Two: Protein Dimerization in Biology. Trends Biochem. Sci. 2004, 29, 618-625.

(10) Gordon, D. E.; Jang, G. M.; Bouhaddou, M.; Xu, J.; Obernier, K.; White, K. M.; O’Meara, M. J.; Rezelj, V. V.; Guo, J. Z.; Swaney, D. L.; Tummino, T. A.; Hüttenhain, R.; Kaake, R. M.; Richards, A. L.; Tutuncuoglu, B.; Foussard, H.; Batra, J.; Haas, K.; Modak, M.; Kim, M.; Haas, P.; Polacco, B. J.; Braberg, H.; Fabius, J. M.; Eckhardt, M.; Soucheray, M.; Bennett, M. J.; Cakir, M.; McGregor, M. J.; Li, Q.; Meyer, B.; Roesch, F.; Vallet, T.; Mac Kain, A.; Miorin, L.; Moreno, E.; Naing, Z. Z. C.; Zhou, Y.; Peng, S.; Shi, Y.; Zhang, Z.; Shen, W.; Kirby, I. T.; Melnyk, J. E.; Chorba, J. S.; Lou, K.; Dai, S. A.; Barrio-Hernandez, I.; Memon, D.; Hernandez-Armenta, C.; Lyu, J.; Mathy, C. J. P.; Perica, T.; Pilla, K. B.; Ganesan, S. J.; Saltzberg, D. J.; Rakesh, R.; Liu, X.; Rosenthal, S. B.; Calviello, L.; Venkataramanan, S.; Liboy-Lugo, J.; Lin, Y.; Huang, X. P.; Liu, Y. F.; Wankowicz, S. A.; Bohn, M.; Safari, M.; Ugur, F. S.; Koh, C.; Savar, N. S.; Tran, Q. D.; Shengjuler, D.; Fletcher, S. J.; O'Neal, M. C.; Cai, Y.; Chang, J. C. J.; Broadhurst, D. J.; Klippsten, S.; Sharp, P. P.; Wenzell, N. A.; Kuzuoglu-Ozturk, D.; Wang, H. Y.; Trenker, R.; Young, J. M.; Cavero, D. A.; Hiatt, J.; Roth, T. L.; Rathore, U.; Subramanian, A.; Noack, J.; Hubert, M.; Stroud, R. M.; Frankel, A. D.; Rosenberg, O. S.; Verba, K. A.; Agard, D. A.; Ott, M.; Emerman, M.; Jura, N.; von Zastrow, M.; Verdin, E.; Ashworth, A.; Schwartz, O.; d'Enfert, C.; Mukherjee, S.; Jacobson, M.; Malik, H. S.; Fujimori, D. G.; Ideker, T.; Craik, C. S.; Floor, S. N.; Fraser, J. S.; Gross, J. D.; Sali, A.; Roth, B. L.; Ruggero, D.; Taunton, J.; Kortemme, T.; Beltrao, P.; Vignuzzi, M.; García-Sastre, A.; Shokat, K. M.; Shoichet, B. K.; Krogan, N. J. A SARS-CoV-2 Protein Interaction Map Reveals Targets for Drug Repurposing. Nature 2020, 583, 459-468..

(11) Lapidus, L. J. Understanding Protein Aggregation from the View of Monomer Dynamics. Mol. Biosyst. 2013, 9, 29-35.

(12) Murthy, A. C.; Dignon, G. L.; Kan, Y.; Zerze, G. H.; Parekh, S. H.; Mittal, J.; Fawzi, N. L. Molecular Interactions Underlying Liquid-liquid Phase Separation of the FUS LowComplexity Domain. Nat. Struct. Mol. Biol. 2019, 26, 637-648.

(13) Molliex, A.; Temirov, J.; Lee, J.; Coughlin, M.; Kanagaraj, A. P.; Kim, H. J.; Mittag, T.; Taylor, J. P. Phase Separation by Low Complexity Domains Promotes Stress Granule Assembly and Drives Pathological Fibrillization. Cell 2015, 163, 123-133.

(14) Vendruscolo, M.; Knowles, T. P. J.; Dobson, C. M. Protein Solubility and Protein Homeostasis: A Generic View of Protein Misfolding Disorders. Cold Spring Harb. Perspect. Biol. 2011, 3, a010454. 
(15) Kato, M.; Han, T. W.; Xie, S.; Shi, K.; Du, X.; Wu, L. C.; Mirzaei, H.; Goldsmith, E. J.; Longgood, J.; Pei, J.; Grishin, N. V; Frantz, D. E.; Schneider, J. W.; Chen, S.; Li, L.; Sawaya, M. R.; Eisenberg, D.; Tycko, R.; McKnight, S. L. Cell-Free Formation of RNA Granules: Low Complexity Sequence Domains Form Dynamic Fibers within Hydrogels. Cell 2012, 149, 753-767.

(16) Borgia, M. B.; Nickson, A. A.; Clarke, J.; Hounslow, M. J. A Mechanistic Model for Amorphous Protein Aggregation of Immunoglobulin-like Domains. J. Am. Chem. Soc. 2013, 135, 6456-6464.

(17) Chiti, F.; Dobson, C. M. Protein Misfolding, Functional Amyloid, and Human Disease. Annu. Rev. Biochem. 2006, 75, 333-366.

(18) Sunde, M.; Serpell, L. C.; Bartlam, M.; Fraser, P. E.; Pepys, M. B.; Blake, C. C. F. Common Core Structure of Amyloid Fibrils by Synchrotron X-Ray Diffraction. J. Mol. Biol. 1997, 273, 729-739.

(19) Luo, F.; Gui, X.; Zhou, H.; Gu, J.; Li, Y.; Liu, X.; Zhao, M.; Li, D.; Li, X.; Liu, C. Atomic Structures of FUS LC Domain Segments Reveal Bases for Reversible Amyloid Fibril Formation. Nat. Struct. Mol. Biol. 2018, 25, 341-346.

(20) Rochet, J. C.; Lansbury, P. T. Amyloid Fibrillogenesis: Themes and Variations. Curr. Opin. Struct. Biol. 2000, 10, 60-68.

(21) Urbanc, B.; Cruz, L.; Yun, S.; Buldyrev, S. V.; Bitan, G.; Teplow, D. B.; Stanley, H. E. In Silico Study of Amyloid $\beta$-Protein Folding and Oligomerization. Proc. Natl. Acad. Sci. U. S. A. 2004, 101, 17345-17350.

(22) Barz, B.; Urbanc, B. Dimer Formation Enhances Structural Differences between Amyloid $\beta$-Protein (1-40) and (1-42): An Explicit-Solvent Molecular Dynamics Study. PLoS One 2012, 7, e34345.

(23) Qiao, Y.; Zhang, M.; Liang, Y.; Zheng, J.; Liang, G. A Computational Study of SelfAssembled Hexapeptide Inhibitors against Amyloid- $\beta$ (A $\beta$ ) Aggregation. Phys. Chem. Chem. Phys. 2016, 19, 155-166.

(24) Carballo-Pacheco, M.; Strodel, B. Advances in the Simulation of Protein Aggregation at the Atomistic Scale. J. Phys. Chem. B 2016, 120, 2991-2999.

(25) Conchillo-Solé, O.; de Groot, N. S.; Avilés, F. X.; Vendrell, J.; Daura, X.; Ventura, S. AGGRESCAN: A Server for the Prediction and Evaluation of "Hot Spots" of Aggregation in Polypeptides. BMC Bioinformatics 2007, 8, 65.

(26) Aggeli, A.; Nyrkova, I. A.; Bell, M.; Harding, R.; Carrick, L.; McLeish, T. C. B.; Semenov, A. N.; Boden, N. Hierarchical Self-Assembly of Chiral Rod-like Molecules as a Model for Peptide $\beta$-Sheet Tapes, Ribbons, Fibrils, and Fibers. Proc. Natl. Acad. Sci. U. S. A. 2001, 98, 11857-11862.

(27) Bucciantini, M.; Giannoni, E.; Chiti, F.; Baroni, F.; Taddei, N.; Ramponi, G.; Dobson, C. M.; Stefani, M. Inherent Toxicity of Aggregates Implies a Common Mechanism for Protein Misfolding Diseases. Nature 2002, 416, 507-511.

(28) Volles, M. J.; Lee, S. J.; Rochet, J. C.; Shtilerman, M. D.; Ding, T. T.; Kessler, J. C.; Lansbury, P. T. Vesicle Permeabilization by Protofibrillar $\alpha$-Synuclein: Implications for the Pathogenesis and Treatment of Parkinson's Disease. Biochemistry 2001, 40, 


\section{2-7819.}

(29) Hwang, W.; Zhang, S.; Kamm, R. D.; Karplus, M. Kinetic Control of Dimer Structure Formation in Amyloid Fibrillogenesis. Proc. Natl. Acad. Sci. U. S. A. 2004, 101, 12916-12921.

(30) Meral, D.; Urbanc, B. Discrete Molecular Dynamics Study of Oligomer Formation by N-Terminally Truncated Amyloid $\beta$-Protein. J. Mol. Biol. 2013, 425, 2260-2275.

(31) Fonda, B. D.; Jami, K. M.; Boulos, N. R.; Murray, D. T. Identification of the Rigid Core for Aged Liquid Droplets of an RNA-Binding Protein Low Complexity Domain. J. Am. Chem. Soc. 2021, 143, 6657-6668.

(32) Mier, P.; Paladin, L.; Tamana, S.; Petrosian, S.; Hajdu-Soltész, B.; Urbanek, A.; Gruca, A.; Plewczynski, D.; Grynberg, M.; Bernadó, P.; Gáspári, Z.; Ouzounis, C. A.; Promponas, V. J.; Kajava, A. V.; Hancock, J. M.; Tosatto, S. C. E.; Dosztanyi, Z.; Andrade-Navarro, M. A. Disentangling the Complexity of Low Complexity Proteins. Brief. Bioinform. 2020, 21, 458-472.

(33) Marcotte, E. M.; Pellegrini, M.; Yeates, T. 0.; Eisenberg, D. A Census of Protein Repeats. J. Mol. Biol. 1999, 293, 151-160.

(34) Simon, M.; Hancock, J. M. Tandem and Cryptic Amino Acid Repeats Accumulate in Disordered Regions of Proteins. Genome Biol. 2009, 10, R59.

(35) Wootton, J. C. Sequences with "unusual" Amino Acid Compositions. Curr. Opin. Struct. Biol. 1994, 4, 413-421.

(36) Toll-Riera, M.; Radó-Trilla, N.; Martys, F.; Albá, M. M. Role of Low-Complexity Sequences in the Formation of Novel Protein Coding Sequences. Mol. Biol. Evol. 2012, 29, 883-886.

(37) Martin, E. W.; Mittag, T. Relationship of Sequence and Phase Separation in Protein Low-Complexity Regions. Biochemistry 2018, 57, 2478-2487.

(38) Cascarina, S. M.; Elder, M. R.; Ross, E. D. Atypical Structural Tendencies among LowComplexity Domains in the Protein Data Bank Proteome. PLoS Comput. Biol. 2020, 16, e1007487.

(39) Ray, M. T.; Tycko, R. Side Chain Hydrogen-Bonding Interactions within Amyloidlike Fibrils Formed by the Low-Complexity Domain of FUS: Evidence from Solid State Nuclear Magnetic Resonance Spectroscopy. Biochemistry 2020, 59, 364-378.

(40) Faltova, L.; Küffner, A. M.; Hondele, M.; Weis, K.; Arosio, P. Multifunctional Protein Materials and Microreactors Using Low Complexity Domains as Molecular Adhesives. ACS Nano 2018, 12, 9991-9999.

(41) Colletier, J. P.; Laganowsky, A.; Landau, M.; Zhao, M.; Soriaga, A. B.; Goldschmidt, L.; Flot, D.; Cascio, D.; Sawaya, M. R.; Eisenberg, D. Molecular Basis for Amyloid- $\beta$ Polymorphism. Proc. Natl. Acad. Sci. U. S. A. 2011, 108, 16938-16943.

(42) Jorgensen, W. L.; Chandrasekhar, J.; Madura, J. D.; Impey, R. W.; Klein, M. L. Comparison of Simple Potential Functions for Simulating Liquid Water. J. Chem. Phys. 1983, 79, 926-935.

(43) Huang, J.; Mackerell, A. D. CHARMM36 All-Atom Additive Protein Force Field: 
Validation Based on Comparison to NMR Data. J. Comput. Chem. 2013, 34, 21352145.

(44) Hess, B.; van der Spoel, D.; Abraham, M. J.; Lindahl, E. On the Importance of Accurate Algorithms for Reliable Molecular Dynamics Simulations. ChemRxiv 2019, 11474583.

(45) Press, W. H.; Teukolsky, S. A.; Vetterling, W. T.; Flannery, B. P. Numerical Recipes 3rd Edition: The Art of Scientific Computing, 3rd ed.; Cambridge University Press: USA, 2007.

(46) Darden, T.; York, D.; Pedersen, L. Particle Mesh Ewald: An N•log(N) Method for Ewald Sums in Large Systems. J. Chem. Phys. 1993, 98, 10089-10092.

(47) Hess, B.; Bekker, H.; Berendsen, H. J. C.; Fraaije, J. G. E. M. LINCS: A Linear Constraint Solver for Molecular Simulations. J. Comput. Chem. 1997, 18, 1463-1472.

(48) NosÉ, S. A Molecular Dynamics Method for Simulations in the Canonical Ensemble. Mol. Phys. 2002, 100, 191-198.

(49) Bussi, G.; Donadio, D.; Parrinello, M. Canonical Sampling through Velocity Rescaling. J. Chem. Phys. 2007, 126014101.

(50) Parrinello, M.; Rahman, A. Polymorphic Transitions in Single Crystals: A New Molecular Dynamics Method. J. Appl. Phys. 1981, 52, 7182-7190..

(51) Lu, J.; Cao, Q.; Hughes, M. P.; Sawaya, M. R.; Boyer, D. R.; Cascio, D.; Eisenberg, D. S. CryoEM Structure of the Low-Complexity Domain of HnRNPA2 and Its Conversion to Pathogenic Amyloid. Nat. Commun. 2020, 11, 4090. 